\title{
Las composiciones de Francesco Antonio Pistocchi (1659- 1726): listado actualizado de su obra
}

The compositions of Francesco Antonio Pistocchi (1659-1726): up-to-date list of his work

As composições de Francesco Antonio Pistocchi (1659-1726): lista atualizada de seu trabalho

DOI: http://dx.doi.org/10.23913/ricsh.v6i11.120

Alejandra Béjar Bartolo

Universidad de Guanajuato, México alejandrabejar@hotmail.com

Fabrizio Ammetto

Universidad de Guanajuato, México Istituto Italiano Antonio Vivaldi, Venezia, Italia

fammetto@hotmail.it

\section{Resumen}

El cantante, compositor y didacta (maestro de canto) Francesco Antonio Pistocchi (Palermo 1659 - Bolonia 1726) fue un músico ampliamente reconocido por sus contemporáneos (Giuseppe Torelli, Pier Francesco Tosi, Johann Joachim Quantz, entre otros) y, hoy en día, es digno de ser considerado entre los mejores compositores de música vocal del Barroco italiano. Aunque no está todavía catalogada de manera sistemática, su producción merece ser ampliamente estudiada, analizada y ejecutada. Hasta la fecha no existe un catálogo exhaustivo que registre de manera puntual toda su obra, por tal razón, en este estudio se presenta por primera vez un listado actualizado de su amplia producción -que incluye óperas, serenatas, oratorios, cantatas, duetos, tercetos, madrigales, misas (o secciones de misa), salmos, responsorios, cánticos, letanías, himnos, antífonas, motetes, lamentaciones, pastorales y secuencias, además de una colección de música instrumental-, en donde se 
especifica la localización de su música que ha llegado hasta nuestros días (actualmente resguardada en alrededor de treinta diferentes bibliotecas de Alemania, Austria, Bélgica, Francia, Italia, Reino Unido y Estados Unidos de América). Cabe hacer hincapié que muchas de las composiciones de Pistocchi se han identificado y enlistado por primera vez en esta investigación.

Palabras clave: fuentes primarias, música vocal, música instrumental, bibliotecas, RISM (Répertoire International des Sources Musicales).

\section{Abstract}

The singer, composer and didact (singing master) Francesco Antonio Pistocchi (1659 Palermo - Bologna 1726) was a musician, widely acknowledged by his contemporaries (Giuseppe Torelli, Pier Francesco Tosi, Johann Joachim Quantz, among others) and, today, it is worthy of being considered among the best composers of vocal music from the Italian Baroque. Although it is not yet listed in a systematic way, their production deserves to be widely studied, analyzed and played. To date there is a comprehensive catalog that register in a timely manner all his work, for this reason, in this study is presented for the first time an updated list of its wide production -que incluye óperas, serenatas, oratorios, cantatas, duetos, tercetos, madrigales, misas (o secciones de Eucharist), salmos, responsorios, cánticos, letanías, himnos, antífonas, motetes, lamentaciones, pastorales y secuencias, además de una colección de música instrumental-, which specifies the location of your music that has survived to this day (currently sheltered in around thirty different libraries of Germany, Austria, Belgium, France, Italy, United Kingdom and United States of America). It should be stressed that many of the compositions of Pistocchi have been identified and listed for the first time in this research.

Key words: primary sources, vocal music, instrumental music, libraries, RISM (Répertoire International des Sources Musicales), International Inventory of Musical Sources. 


\section{Resumo}

O cantor, compositor e (professor de canto) Didacta Francesco Antonio Pistocchi (Palermo 1659 - Bologna 1726) foi um músico amplamente reconhecido por seus contemporâneos (Giuseppe Torelli, Pier Francesco Tosi, Johann Joachim Quantz, entre outros) e, hoje, é digno de ser considerado um dos melhores compositores da música vocal do barroco italiano. Embora ainda não catalogado sistematicamente, produção merece ser amplamente estudado, analisado e executado. Até à data não existe catálogo exaustivo que registra em tempo hábil toda a sua obra, por essa razão, este estudo apresenta pela primeira vez uma atualizou seu extensa lista de produção que inclui óperas, serenatas, oratórios, cantatas, duetos, trios , Madrigals, massas (ou seções de massa), salmos, responsórios, hinos, ladainhas, hinos, antífonas, motetos, lamentações, pastorais e seqüências, assim como uma coleção de música instrumental, onde a localização da sua música especifica que que chegou a nossos dias (atualmente abrigados em cerca de trinta bibliotecas diferentes na Alemanha, Áustria, Bélgica, França, Itália, Reino Unido e Estados Unidos da América). Deve-se ressaltar que muitas das composições Pistocchi foram identificados e listados pela primeira vez nesta pesquisa.

Palavras-chave: fontes primárias, música vocal, música instrumental, bibliotecas, RISM (repertório internacional des Sources Musicales).

Fecha Recepción: Junio 2016 Fecha Aceptación: Noviembre 2016

\section{Introducción}

¿Es posible que un destacado músico del período barroco sea al día de hoy prácticamente desconocido, mientras que en su época era considerado por sus contemporáneos (y también por sus sucesores inmediatos) un gran artista? En el caso del niño prodigio, cantante, compositor y didacta italiano -fundador de una de las primeras escuelas de canto en ItaliaFrancesco Antonio Pistocchi (Palermo 1659 - Bolonia 1726), apodado “il Pistocchino”, la respuesta es (lamentablemente) afirmativa. De hecho, los diccionarios musicológicos internacionales más importantes -en orden de aparición, el Dizionario enciclopedico 
universale della musica e dei musicisti (Basso, 1985-1988), The New Grove Dictionary of Music and Musicians (Sadie, 2001), Die Musik in Geschichte und Gegenwart (Finscher, 1994-2007)- dedican solo pocas líneas a este músico y, en ciertos casos, las informaciones relativas a su vida resultan contradictorias (incluso lagunosas), o están citadas solamente en uno o dos de los tres diccionarios. Las composiciones musicales de Pistocchi que son mencionadas en estos diccionarios son solo una parte de su notable producción. ${ }^{1} \mathrm{La}$ biografía más actualizada sobre Pistocchi se encuentra en el Dizionario Biografico degli Italiani (Lora, 2015) y, sobre todo, en Béjar Bartolo, 2015 (pp. 3-11).

Pistocchi fue muy estimado por sus extraordinarios méritos artísticos, como demuestran algunas afirmaciones de eminentes músicos contemporáneos a él. El 27 de marzo de 1700, el célebre violinista y compositor Giuseppe Torelli (1658-1709) escribió desde Viena al compositor Giacomo Antonio Perti (1661-1756), maestro de capilla en la Basílica de San Petronio en Bolonia:

«Circa la stima del caro Pistochino qui in Vienna gli giuro che è grandis[si]ma $\mathrm{p}[\mathrm{er}]$ ché toltone la virtù grande del cantare, lui è stimato assais[si]mo nelle sue compositioni, che veram[en]te riusciscono e studiose e vaghe estraordinariam[en]te e non vedo l'ora che lei le veda, e le senta, che so che ancora lei concor[d]erà dispassionatam[en]te con l'opinione e mia e di tutti, che intendono la musica» ${ }^{2}$ (carta de Torelli a Perti, en $I-B c$, P.143.054.a).

En 1723, el renombrado cantante, maestro y compositor Pier Francesco Tosi (ca. 1654 1732) - en el capítulo «Dell'Arie» de su texto Opinioni de' cantori antichi, e moderni, o sieno Osservazioni sopra il canto figurato- opinaba sobre Pistocchi:

1 Las listas de las composiciones de Pistocchi presentes en los tres diccionarios musicales antes mencionados son parciales (Chiarelli, 1988; Dubowy, 2007; Schnoebelen, 2001).

2 Traducción: «Sobre de la estima del querido 'Pistoc[c]hino' aquí en Viena le juro que es grandísima, ya que además de la gran virtud en el cantar, es sumamente estimado por sus composiciones, que ciertamente son extraordinarias tanto en la elaboración como en la inspiración, y no veo la hora que usted las vea y las escuche; yo sé que usted estará totalmente de acuerdo con mi opinión y con la de todos los demás que entienden la música». 
«[...] chi sì sa cantare trova sul Tempo congruenza di sito che serva agli abbellimenti dell'arte senza inventare, né mendicar pause. [...] il primo fu il Signor Pistocchi Musico il più insigne de' nostri, e di tutti i tempi, il di cui nome si è reso immortale per essere stato egli l'unico Inventore d'un gusto finito, e inimitabile, e per aver insegnato a tutti le bellezze dell'Arte senza offendere le misure del Tempo» ${ }^{3}$ (Tosi, 1723, p. 65).

Con estas palabras, Olivo Penna -historiador de la muy célebre Academia Filarmónica de Bolonia, de la cual Pistocchi fue nombrado en dos ocasiones «Principe» (cargo de mayor rango)- definió en 1736 a Pistocchi (diez años después de su muerte) en su monumental Cronologia, o sia Istoria Generale di questa Accademia, como:

«Compositore di Musica eccellente, e Cantante de più rari che abbia avuto l'Europa, e per tale ammirato anche dagli Oltramontani che ne hanno fatto (come in Londra) la memoria gloriosa fra gli uomini famosi e tale l'hanno sospirato non solo i di lui bravi scolari riusciti in poco tempo, ma quanti l'hanno conosciuto» $^{4}$ (Penna, 1736, vol. I, p. 259).

En 1752, el célebre flautista, compositor y teórico musical Johann Joachim Quantz (16971773) escribió sobre Pistocchi en su Versuch einer Anweisung die Flöte traversiere zu spielen:

3 Traducción: «[...] quien sí sabe cantar encuentra en el Tempo congruencia de lugar que sirva a los adornos del arte sin inventar, ni mendigar pausas. [...] el primero fue el Señor Pistocchi, el Músico más insigne de nuestros y de todos los tiempos, cuyo nombre se volvió inmortal por haber sido el único inventor de un gusto refinado e inimitable, y por haber enseñado a todos las bellezas del Arte sin ofender las medidas del Teтро».

4 Traducción: «Compositor de Música excelente, y Cantante de los más raros que había habido en Europa, y por eso admirado también en el Norte de los Alpes, [cualidades] que hicieron (como en Londres) su gloriosa memoria entre los hombres famosos, y como tal lo han querido no solamente sus alumnos destacados que llegaron a ser buenos en poco tiempo, pero también todos los que lo han conocido». 
«Dacché Pistocchi ha aperte sulla fine dell'andato secolo, le sue scuole da cantare, ed doppo che ha principiato dare al mondo tanti eccellenti cantori, l'arte del cantare è anche giunta al suo supremo grado nel tempo de' primi trent'anni del secolo corrente, e molti cantori, che meritano raggionevolmente la taccia di ragguardevoli, hanno fatto conoscere, ed hanno eseguito quasi tutto quello che si può far sentire di commotivo, e di maraviglioso colla voce umana; in quante occasioni non hanno bravi Compositori pigliato da ciò motivo per perfezionare di mano in mano la composizione delle opere cantabili? Corelli, ed i suoi successori hanno molto lodevolmente fatto in maniera, di pareggiarli nel merito in riguardo alla Musica instrumentale» ${ }^{5}$ (Quantz 1752, § 56). ${ }^{6}$

Así pues, para descubrir la calidad artística y la importancia histórica de la música de Francesco Antonio Pistocchi es necesario, primeramente, identificar un listado actualizado de su obra.

\section{Método}

Antes que nada, para la realización de la presente investigación ha sido fundamental la consulta de varios catálogos de fuentes musicales en línea (además de la literatura secundaria sobre Pistocchi): Catalogo nazionale dei manoscritti musicali redatti fino al 1900. Milano, Biblioteca Nazionale Braidense, Ufficio Ricerca Fondi Musicali (BRAIDENSE); "Clori” - Archivio della Cantata italiana (ClORI); "Corago” (CORAGO); 9

5 Traducción: «A partir de que Pistocchi abrió, al final del siglo pasado, sus escuelas de canto, y después de ofrecer al mundo muchos cantantes excelentes, el arte del cantar ha llegado también al máximo grado en el tiempo de los primeros treinta años del siglo actual, y muchos cantantes, que merecen con razón la mención de sobresalientes, hicieron conocer y ejecutaron casi todo lo que se puede hacer escuchar que conmueva y que maraville con la voz humana; ¿en cuántas ocasiones buenos Compositores no aprovecharon de mano en mano esta habilidad para perfeccionar la composición de las óperas cantábiles? Corelli, y sus sucesores loablemente lo hicieron de la misma manera para igualarlos en la Música instrumental».

6 Presentado aquí en una traducción italiana -manuscrita y anónima- elaborada antes de 1784 y resguardada en la biblioteca privada del Padre Martini, alumno de Pistocchi (I-Bc, ms. H 75, cc. 131r-290r).

7 En línea: <http://www.urfm.braidense.it/cataloghi/>.

8 En línea: <http://www.cantataitaliana.it/>. 
“OPAC SBN” - Catalogo del Servizio Bibliotecario Nazionale [italiano] (OPAC SBN); ${ }^{10}$ RISM (Répertoire International des Sources Musicales) - Online Catalogue of Musical Sources (RISM). ${ }^{11}$

Las composiciones de Pistocchi recabadas de dichos bancos de datos han sido analizadas y divididas por géneros. Las obras fechadas (óperas, oratorios, etc.) han sido enlistadas en orden cronológico, mientras que las no fechadas (cantatas, duetos, tercetos, etc.) aparecen en orden alfabético. Después del título de cada ópera, serenata y oratorio se ha especificado: nombre del autor del texto (entre paréntesis); género (en su idioma original) o número de voces, según sea el caso; ciudad (escrita en su idioma original), lugar (también escrito en su idioma original) y fecha de estreno. Para las otras composiciones vocales -cantatas, duetos, tercetos, madrigales, misas (o secciones de misa), salmos, responsorios, cánticos, letanías, himnos, antífonas, motetes, lamentaciones, pastorales, secuencias- se ha especificado entre paréntesis el tipo de voz (si es desconocido se ha escrito dentro del paréntesis un signo de interrogación) y la fecha de ejecución (si se conoce). Al final se han añadido las siglas RISM relativas a los lugares donde está preservada la música (hasta hoy identificada) y su signatura; también para los textos poéticos escritos por el mismo Pistocchi se han proporcionado las informaciones relativas a la localización de las fuentes. En el caso de sus colecciones publicadas en vida (Capricci puerili, op. I; Scherzi musicali, [op. II]; Duetti e terzetti, op. III) han sido separadas las fuentes impresas de las manuscritas (si existen).

Se han utilizado las siguientes abreviaturas:

$\mathrm{B}=$ Basso / Bajo (voz); bc = continuo; $\mathrm{C}=$ Alto / Contralto $(\mathrm{voz}) ; \mathrm{c} . / \mathrm{cc} .=$ carta/e, folio/s; ca. $=$ cerca; col. $/$ cols. $=$ columna $/$ columnas; no. / nos. $=$ número/s; ob = oboe/s; op. $/$ opp . = opus / opera (obra / obras); org = órgano; pág. / págs. = página/s; $\S$ = párrafo; $\mathrm{S}=$ Canto / Soprano (voz); T = Tenore / Tenor (voz); viol = violón; vl = violín/violines; vla = viola/s; vlc $=$ violonchelo/s; vol. / vols. $=$ volumen / volúmenes.

9 En línea: <http://corago.unibo.it/>.

10 En línea: <http://www.sbn.it/>.

11 En línea: <http://www.rism.info/>. Las abreviaturas de las bibliotecas citadas más adelante son aquellas utilizadas a nivel internacional por el RISM. 


\section{Resultados}

Los resultados de la presente investigación han sido la recopilación y localización (en el caso de las obras cuya música ha llegado hasta nuestros días) de alrededor de 150 composiciones de Pistocchi, ${ }^{12}$ varias de las cuales se han identificado por primera vez.

\section{ÓPERAS: $:^{13}$}

- Il Leandro (Camillo Badovero), ${ }^{14}$ “dramma per musica”, Venezia, Teatro alle Zattere, 5 de mayo de 1679 (con el título Gli amori fatali, ${ }^{15}$ Venezia, Teatro S. Moisè, enero de 1682). Arias en $B-B c(?)$ y $I-V q s(?)^{16}$ [RISM: deest $] .{ }^{17}$

${ }^{12}$ En este sentido no nos sorprende la consideración del célebre músico y musicólogo inglés Charles Burney (1726-1814) al escribir: «Pistocchi seems a more correct contrapuntist than the generality of opera singers whom the demon of composition seizes at a period of their lives» (traducción: Pistocchi parece mucho más experto en el contrapunto que la generalidad de los cantantes de ópera a quienes el demonio de la composición se apodera en un período de sus vidas); ver Burney, 1789, vol. 4, p. 114.

13 Además de las óperas aquí presentadas, en $I-B c$ (colocación: Lo.6808) está conservado el libreto del "dramma per musica" Nerone fatto Cesare (texto de Matteo Noris) representado en el Teatro S. Angelo de Venezia durante el carnaval de 1715. Este drama consiste en un "pasticcio" -catalogado entre las óperas de Antonio Vivaldi como RV 724 por Peter Ryom (Ryom, 2007, pp. 452-453)-, ya que en la parte posterior de la portada del libreto aparece una larga lista de arias de diferentes maestros: «Antonio Pollarolo, il Vivaldi, Francesco Gasperini, Giacomo Perti, l'Orlandini, Antonio Carli, il Pistocchi». Pistocchi es el autor de la música (perdida) del aria «Lusinga il Dio d'Amor» (III:11), como menciona la fuente: «del q. D. Pistoccho».

${ }^{14}$ El letrado veneciano Giovanni Carlo Bonlini (1673-1731) escribió que Il Leandro -cuya música fue compuesta por «Franc. Ant. Pistochini»- «fu rappresentato con Figure di Legno, cantando i Musici dietro le Scene in una Casa su la Riva, o Fondamenta detta le Zattare [sic], verso i Ogni Santi» (Bonlini, 1730, p. 90), o sea fue representado con figuras de madera, con los músicos cantando detrás de las escenas en una casa a orillas del río.

${ }^{15}$ Bonlini escribió que «questo Drama [Gli amori fatali] non ha quasi altra mutazione, che il Nome solo, da che l'anno 1679, col Titolo di Leandro fu rappresentato alle Zattare [sic] con Figure, come ora si rappresenta anche in questo Teatro» (Bonlini, 1730, p. 97), o sea este drama fue representado exactamente igual que el de 1679 a excepción del cambio en el nombre.

16 Según Dubowy, 2007, col. 637, aunque no especifica la signatura.

17 La expresión deest (del latín de, negación, y est, estar) significa que carece del número RISM. 
- Il Girello ${ }^{18}$ (Filippo Acciaioli), “dramma per musica”, Venezia, Teatro S. Moisè, enero de 1682.

- Il Narciso (Apostolo Zeno), "pastorale per musica”, Ansbach, Hoftheater, marzo de 1697. Partitura en GB-Lbl, R.M.22.m.25. [RISM: 800252042]. Partichela del «Basso Continuo e Violoncello» en $G B-L b l$, R.M.22.m.26.(16.) [RISM: 800252059].

- Le pazzie d'amore e dell'interesse (Pistocchi), "idea drammatica per musica", Ansbach, Hoftheater, 16 de junio de 1699. Libreto en I-Rn (faltan las cc. A3-6). Música perdida. - Le risa di Democrito (Nicolò Minato), “dramma per musica”, Wien, Hoftheater, 17 de febrero de 1700. Partitura (solamente II Acto) en A-Wn, Mus.Hs.16015 y Mus.Hs.19242 (no. 104) [RISM: deest], y en US-Wc, M1500.P66 R4 (Case) [RISM: 900011225].

- I rivali generosi (Apostolo Zeno), “dramma per musica” en colaboración con Clemente Monari y Giovanni Maria Cappelli (Pistocchi compuso el II Acto), Reggio Emilia, Teatro Pubblico, abril de 1710. Música perdida.

\section{SERENATAS:}

- La pace tra l'armi (Pistocchi), serenata, Ansbach, 5 de septiembre de 1700. Música perdida.

\section{ORATORIOS: ${ }^{19}$}

- Il martirio di S. Adriano (Silvio Stampiglia), a cuatro voces, Modena, 26 de agosto de 1692. Partitura en I-MOe (?) ${ }^{20}$ [RISM: deest].

${ }^{18}$ No obstante Leonida Busi no considera Il Girello una ópera de Pistocchi (Busi, 1891, pp. 148-149), Bonlini escribió que este Drama -cuya música fue compuesta por «Franc. Ant. Pistochino»- fue «rappresentato con Figurine di Cera, fu prima più volte recitato altrove» (Bonlini, 1730, pp. 95-96), o sea fue representado con figurillas de cera y antes en otros lugares.

${ }^{19}$ Los cinco oratorios mencionados en este apartado son auténticamente de Pistocchi. Sin embargo, sin especificar la fuente, Lora, 2015, adjudica también a Pistocchi el oratorio La spiegazione de' sogni di Gioseppe ebreo (Rimini, 1716). Finalmente, el oratorio La fuga di Santa Teresa (texto de E. Manfredi), Forlì, 1717, es una opera dubia (Busi, 1891, pp. 178-179).

20 Según Dubowy, 2007, col. 637, aunque no especifica la signatura. 
- Maria Vergine addolorata (?), Ansbach, 1698. Aria de la «Maddalena»E che farei misera me! en $A-W n$, SA.68.D.88 (publicada por Charles Burney) ${ }^{21}$ [RISM: deest].

- Davide (?), a cinco voces, Firenze, Compagnia dell'Arcangelo Raffaello detta La Scala, 31 de diciembre de 1712; Bologna, Oratorio de' Filippini detti della Madonna di Galliera, 19 de marzo de 1721. Música perdida.

- Il sacrificio di Gefte (Giovanni Battista Neri), a cuatro voces, Bologna, Oratorio de' Filippini detti della Madonna di Galliera, 1 de noviembre de 1720. Parte del Bajo («Eleazaro») en I-Bof, ms 22.a [RISM: 850034907].

- I pastori al presepe (Stefano Pallavicini), Bologna, Oratorio de' Filippini detti della Madonna di Galliera, 25 de diciembre de 1721. Música perdida.

\section{MÚSICA INSTRUMENTAL:}

- Capricci puerili variamente composti e passeggiati in 40 modi sopra un basso d'un balletto [...] per suonarsi nel clavicembalo, arpa, violino et altri stromenti, op. I, Bologna, Giacomo Monti, 1667. ${ }^{22}$ Impresión: I-Bc, BB.157 [RISM A/I: P 2456].

\section{CANTATAS $^{23}\left(\right.$ Y ARIAS): ${ }^{24}$}

21 Burney, 1789, vol. 4, pp. 114-115 y 121.

22 Edición crítica en Béjar Bartolo, 2010, pp. 105-133.

${ }^{23}$ Una colección de «Cantate di Fran. ${ }^{\text {co }}$ Ant. ${ }^{\circ}$ Pistocchi op. 1» (Bologna, Marino Silvani, 1698) -hoy perdidaestá registrada en el catálogo del editor musical y compositor boloñés Giuseppe Antonio Silvani (Silvani, 1724, no. 159) y mencionada también en Mischiati, 1984, no. XIX. No es claro por qué Pistocchi autorizó la impresión, también en Bolonia, de estas cantatas como "op. I", siendo que en 1667 ya se habían impreso sus Capricci puerili, op. I: una posible explicación es que no se trataba del "op. I" de Pistocchi, si no del “op. I” de sus cantatas. No obstante Francesco Lora (Lora, 2015) supone que la colección holandesa de los Scherzi musicali (Amsterdam, Estienne Roger, [1698]) de Pistocchi sea una reimpresión "no autorizada" de una impresión anterior de Marino Silvani (¿acaso la colección perdida de «Cantate [...] op. 1»?), es extremadamente probable que el contenido de las cantatas perdidas op. I de Pistocchi sea distinto al de los Scherzi musicali: por un lado, la impresión de los Scherzi musicali no incluye solamente cantatas -como menciona el título de la colección perdida-, sino también arias y duetos; y por otro lado, el musicólogo holandés Rudolf Rasch no excluye que Pistocchi pudo haber acompañado a Giuseppe Torelli a Ámsterdam entre 1697 y 1698, para establecer contactos directos con el editor Estienne Roger en función de la publicación de sus Scherzi musicali (Rasch, 2001). 
- Accompagna il mio partir (?). I-Fc, D.II.568 [RISM: deest].

- Amorosa pastorella (S). GB-Lbl, Add. 31594 [RISM: 806046097].

- Amorosa violetta (S), [op. II], ${ }^{25}$ no. 3. Impresión: I-Bc, BB.158 [RISM A/I: P 2458].

Manuscritos: D-Bsa, SA 1278 (8) [RISM: 469127808]; $D$-W, Cod. Guelf. 266 Mus.

Hdschr. (Nr. 48) ${ }^{26}$ [RISM: 451508489]; GB-Lbl, Add. 34053 [RISM: 806043010].

- Bacia e rendimi il mio core (?). I-Fc, D.II.568 [RISM: deest].

- Beaux yeux d'Amarillis (Aire) (S), aria, [op. II], no. 10. Impresión: I-Bc, BB.158 [RISM A/I: P 2458]. Manuscritos: D-Bsa, SA 1278 (13) [RISM: 469127813]; GB-Lbl, R.M.22.m.27 [RISM: 800252060].

- Bella Irene, cara vita (?). I-Fc, D.II.568 [RISM: deest].

- Bel sospiro che dal seno (C). GB-Lbl, Add. 14228 [RISM: 806155088].

- Caro il mio caro amor (S), aria. GB-Lam, MS 143 [RISM: 800159348].

- Che colpa questo core (S). GB-Lbl, Add. 14226 ${ }^{27}$ [RISM: 806155017]; I-Nc, Cantate 228(3) [RISM: deest].

- Chiari fonti bei fiori (S). D-MÜs, SANT Hs 859 (Nr. 16) [RISM: 451002504].

- Cinta dal velo usato (C). F-Pn, RES VMC MS-81-5 ${ }^{28}$ [RISM: deest]; GB-Lbl, Add. 31594 [RISM: 806046095].

${ }^{24}$ Las que siguen son las cantatas que aparecen en el RISM y/o en catálogos de diferentes bibliotecas. Hay algunas composiciones constituidas por una sola 'aria' o 'recitativo-aria', y de otras se desconoce si forman parte de una cantata o si son solo piezas independientes. La lista está formada de acuerdo al incipit alfabético del texto (se señala solamente la primera línea). Las cantatas Non veduta ancor m'impiaga y Tratto qui da un cieco Nume -en cuyos manuscritos resguardados en I-Rli (Musica M 15/3) aparece el nombre de «Pistocchino»- son en realidad composiciones de Giacomo Carissimi (como se puede ver en las otras fuentes de las mismas cantatas resguardas en $D$-MÜs, SANT Hs 904 (Nr. 3) y SANT Hs 904 (Nr. 7), respectivamente): Pistocchi fue probablemente el intérprete de estas dos cantatas.

25 En su totalidad, las doce composiciones del “op. II" son parte de la colección impresa Scherzi musicali (Amsterdam, Estienne Roger, [1698]), que contiene seis cantatas, dos duetos, dos composiciones vocales en francés y otras dos en alemán. Por el hecho de que en 1698 el editor Marino Silvani publicó en Bolonia una colección de cantatas op. I de Pistocchi, los Scherzi musicali son hoy en día identificados, en la literatura secundaria internacional, como “op. II" (y no como “op. I").

${ }^{26}$ En esta fuente la cantata está transcrita una tercera abajo (en Fa mayor) y la parte vocal es para Alto.

27 En esta fuente la cantata es anónima.

28 En esta fuente la cantata es para Soprano. 
- Come Dorinda, come (S). I-Mc, Noseda O.31.13 [RISM: deest].

- Come piuma dal vento agitata (S). I-Fc, D.II.569 [RISM: deest]; F-Pn, RES VMC MS81-4 [RISM: deest].

- Con dolce mormorio (S). I-Bc, DD.44 [RISM: deest].

- Cupido, bleib’ mir doch vom Leibe (C), aria, [op. II], no. 11. Impresión: I-Bc, BB.158 [RISM A/I: P 2458]. Manuscritos: D-Bsa, SA 1278 (15) [RISM: 469127815]; GB-Lbl, R.M.22.m.27 [RISM: 800252060].

- Dal grand'occhio del Cielo (S) [¿incompleta?]. I-Nc, Cantate 228(2) [RISM: deest].

- Dissi un giorno al Dio d'amore (?), aria. I-Fc, F.I.21 [RISM: deest].

- Dolorosa partenza, oh Dio, che l'alma (C), [op. II], no. 4. Impresión: I-Bc, BB.158 [RISM A/I: P 2458]. Manuscritos: D-B, Mus.ms. 30186 ${ }^{29}$ [RISM: 455031390]; D-Bsa, SA 1278 (9) [RISM: 469127809].

- Dormia un di fra l'erbe Clori (S). F-Pn, RES VMC MS-81-2 [RISM: deest].

- Ecco il sole ceppi di gelo (S). D-B, Mus.ms. 30197 [RISM: 455031458]; D-B, Mus.ms. 30197 [455031478].

- E mi lasciasti ingrata(S). I-Mc, Noseda O.31.14 [RISM: deest].

- Filomena che sì bella (S). I-PAc, (?) ${ }^{30}$ [RISM: deest].

- Fuggi Fileno fuggi (S). I-Nc, Cantate 228(1) [RISM: deest].

- Gelsomino, Il / Gelsomino ridente (S). I-Nc, Cantate 228(5) [RISM: deest].

- Gemma cara adorata (S). D-MÜs, SANT Hs 3221 [RISM: 451017512].

- Il giglio e la viola (S). D-MÜs, SANT Hs 3222 [RISM: 451017513].

- In su la piaggia aprìca (S), [op. II], no. 1. Impresión: I-Bc, BB.158 [RISM A/I: P 2458]. Manuscritos: $D-B s a$, SA 1278 (6) [469127806]; F-Pn, RES VMC MS-81-3 [RISM: deest].

- Io che lontan dal core (S). GB-Lam, MS 127 [RISM: 800092765].

- Je me fais un plaisir extrême (Gigue) (C), aria, [op. II], no. 9. Impresión: I-Bc, BB.158 [RISM A/I: P 2458]. Manuscritos: D-Bsa, SA 1278 (12) [RISM: 469127812]; GB-Lbl, R.M.22.m.27 $7^{31}$ [RISM: 800252060].

29 En esta fuente la cantata está transcrita una quinta arriba (en Mi menor) y la parte vocal es para Soprano.

30 OPAC SBN no especifica la signatura. 
- Lilla convien partire (C), aria. I-Tn, Giordano 41 [RISM: deest].

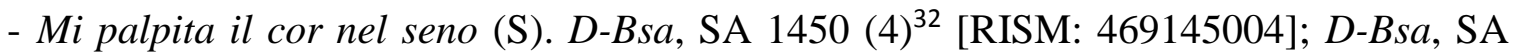
1853 (7) ${ }^{33}$ [RISM: 469185307]; GB-Lgc, G. Mus. 362 (vol. II) [RISM: 806156977]; $G B-L g c$, G. Mus. 362 (vol. II) [RISM: 806156993].

- Ninfe e pastorine i vostri amori (C). D-MÜs, SANT Hs 3223 [RISM: 451017514].

- Non più pene al cor io/mi sento (S). A-Wn, E.M. 178 34 [RISM: 600241072]; D-MÜs, SANT Hs 861 (Nr. 12) $)^{35}$ [RISM: 451002539]; GB-Lbl, Add. $34053^{36}$ [RISM: 806043008].

- O delizia degl'orti (C). F-Pn, RES VMC MS-81-7 [RISM: deest]; GB-Lbl, Add. 31503 [RISM: 806045614]; I-Bsp, Lib. P. 62 [RISM: deest].

- Oh sempre al tuo pastore (C). I-Bc, DD.48 [RISM: deest].

- O placatevi, empie stelle (?), aria. I-MOe, Mus. G. 251 [RISM: deest].

- Pallidetta viola (C). F-Pn, RES VMC MS-81-9 ${ }^{37}$ [RISM: deest]; GB-Lbl, Add. 14228 [RISM: 806155094]; GB-Lbl, Add. 34053 ${ }^{38}$ [RISM: 806043009]; I-Gl, A.7.16 $(\mathrm{A} .1 .5)(4)^{39}$ [RISM deest]; I-PAVu, Ms. Ticinesi $696^{40}$ [RISM: deest].

- Per consolar mie pene (S). A-Wn, E.M. 178 [RISM: 600241073].

- Per te forse mio ben (C). D-SHs, Mus B1:141 [RISM: 250004726]; GB-Lbl, Add. $62102^{42}$ [RISM: 806251381]; I-Pca, D.I.1367 ${ }^{43}$ [RISM: deest].

31 Esta fuente se titula «Una Opera del Sigr. | Pistochi» e incluye las dos composiciones en francés y las dos en alemán.

32 En esta fuente la cantata es para Alto.

33 En esta fuente aparece solamente la aria inicial (registrada como «Mi palpita il cor») sin la parte del bajo.

34 En esta fuente el título es «Non più pene al cor io sento».

35 En esta fuente el título es «Non più pene al cor mi sento».

36 En esta fuente el título es «Non più pene al cor».

37 En esta fuente la cantata es para Soprano.

38 En esta fuente la cantata es para Soprano.

39 En esta fuente la cantata está atribuida erróneamente al «Sig. ${ }^{r}$ [Carlo Francesco] Cesarini» (1666-1741).

40 En esta fuente la cantata es para Soprano. No obstante OPAC SBN no menciona esta cantata, la información se recaba de Dellaborra-Peroni, 2008, pp. 23, 47, 52, 142, 173, 174.

41 En esta fuente la cantata - titulada «Per te forse mio ben amor face non ha»- se atribuye a «[?] Mancini».

42 En esta fuente la cantata es para Soprano. 
- Per un volto di gigli e di rose (S). A-Wn, E.M. 178 [RISM: 600241082]; F-Pn, RES VMC MS-81-6 [RISM: deest]; GB-Lbl, Add. 31503 ${ }^{44}$ [RISM: 806045607]; GB-Lbl, Add. $34053^{45}$ [RISM: 806043007]; GB-Lgc, G. Mus. 362 (vol. I) [RISM: 806156968]; I-Bc, V.292 [RISM: deest].

- Pien di rugiade il seno del sole (C), aria. GB-Ob, Ms. Mus. e.11 [RISM: 800273998].

- Pria mancare o bella Clori (S). F-Pn, RES VMC MS-81-8 [RISM: deest]; GB-Lbl, Add. 34053 [RISM: 806043000].

- Prigioniero d'un bel volto (C), aria. GB-Ob, Ms. Mus. e.11 [RISM: 800273997].

- Pur ch'io cada incenerito (?), aria. I-MOe, Mus. G. 251 [RISM: deest].

- Quand'io vidi il caro volto (C), aria. I-A(?), ${ }^{46}$ Mss. N. 315/5 [RISM: deest].

- Quanto è dolce a questo core (C), [op. II], no. 5. Impresión: I-Bc, BB.158 [RISM A/I: P 2458]. Manuscritos: D-Bsa, SA 1278 (10) [RISM: 469127810$]$ ] I-Fc, D.II.569 [RISM: deest].

- Quanto piace agl'occhi miei (S). GB-Lbl, Add. 14215 [RISM: 806154677].

- Quei momenti che vivo (S). I-Nc, Cantate 228(6) [RISM: deest].

- Se credi a quel bel volto (C). I-Pca, D.I.1367 [RISM: deest].

- Se m’impiaga pupilla vaga (S), [op. II], no. 2. Impresión: I-Bc, BB.158 [RISM A/I: P 2458]. Manuscritos: D-Bsa, SA 1278 (7) [RISM: 469127807]; I-Fc, D.II.569 [RISM: deest].

- Semplice farfalletta avezza al lume (S). GB-Lbl, Add. 14215 [RISM: 806154681].

- Sovra un bel poggio (S). D-B, Mus.ms. 30197 [RISM: 455031469].

- Sprezzarla è ingratitudine (?), aria. I-MOe, Mus. G. 251 [RISM: deest].

- Su la riva del mar tutto dolente (B), [op. II], no. 6. Impresión: I-Bc, BB.158 [RISM A/I: P 2458]. Manuscrito: D-Bsa, SA 1278 (11) [RISM: 469127811].

- Tortorelle imprigionate (C). D-MÜs, SANT Hs 861 (Nr. 11) [RISM: 451002538]; GBLbl, Add. 31503 [RISM: 806045619].

${ }^{43}$ En esta fuente el título es «Per te forsi [sic] mio ben».

44 En esta fuente la cantata es para Contralto.

45 En esta fuente el título es «Per un volto di gigli».

${ }^{46} \mathrm{La}$ respectiva ficha presente en BRAIDENSE reporta la sigla RISM incompleta «I-A», que no da la posibilidad de localizar exactamente la partitura manuscrita de esta composición. 
- Viver lungi dal bel (S). I-Nc, Cantate 228(4) [RISM: deest].

- Zorn mit dem Geliebten führen (S), aria, [op. II], no. 12. Impresión: I-Bc, BB.158 [RISM A/I: P 2458]. Manuscritos: D-Bsa, SA 1278 (16) [RISM: 469127816]; GB-Lbl, R.M.22.m.27 [RISM: 800252060].

\section{DuETOS: ${ }^{47}$}

- Amor, non fai per me (SC), op. III, ${ }^{48}$ no. 10 . Impresión: $I-B c$, BB.159; I-Fc, $(?)^{49}[\mathrm{RISM}$ A/I: P 2457].

- Chi non ama la catena (SC), op. III, no. 8. Impresión: I-Bc, BB.159; I-Fc, (?) [RISM A/I: P 2457].

- Di dolcezze grate al cor (SC), op. III, no. 6. Impresión: I-Bc, BB.159; I-Fc, (?) [RISM A/I: P 2457]. Manuscrito: GB-Lbl, R.M.23.k.6.(2.) [RISM: 800260707].

- Dolcissima speranza (SC), op. III, no. 2. Impresión: I-Bc, BB.159; I-Fc, (?) [RISM A/I: P 2457].

- Frena, Amor, gli sdegni tuoi (SC), [op. II], no. 8. Impresión: I-Bc, BB.158 [RISM A/I: P 2458]. Manuscrito: $B-B r$, Ms II 3951 Mus Fétis 2430 [RISM: 700006573 ].

- Il languir tra lacci involto (SB). D-AN, VIg $57^{50}$ [RISM: 453012067].

- Lontanto dal mio bene (SC). GB-Lbl, Add. 31491 [RISM: 806935309].

- Luci care che ferite (SS). D-AN, VIg 57 [RISM: 453012068].

- M'incatenò Cupido (SC), op. III, no. 7. Impresión: I-Bc, BB.159; I-Fc, (?) [RISM A/I: P 2457]. Manuscritos: GB-Lbl, Add. 5335 [RISM: 806034570]; GB-Lbl, Add. 5056 [RISM: 800265630]; GB-Lbl, Add. $31492^{51}$ [RISM: 806045555].

47 El Dueto de “Bertoldo”-mencionado solamente en Schnoebelen, 2001, p. 791- no es una composición de Pistocchi, sino un dueto de Francesco Piticchio (RISM: 455027444).

48 En su totalidad, las doce composiciones del "op. III" son parte de la colección impresa Duetti e terzetti (Bologna, Marino Silvani, 1707), que contiene diez duetos y dos tercetos.

49 OPAC SBN no especifica la signatura.

50 Todos los duetos en $D-A N$ son anónimos: la atribución a Pistocchi es sugerida por Kai Wessel, quién también ha curado la edición de los mismos (Wessel, 2004).

51 En esta fuente (con fecha de 1786) las partes vocales del dueto son para Soprano y Tenor. 
- Nel bel volto del mio diletto (SC), op. III, no. 4. Impresión: I-Bc, BB.159; I-Fc, (?) [RISM A/I: P 2457].

- Non curo d'aver piacer (SC), op. III, no. 9. Impresión: I-Bc, BB.159; I-Fc, (?) [RISM A/I: P 2457].

- Non si chiama penar (SS), [op. II], no. 7. Impresión: I-Bc, BB.158 [RISM A/I: P 2458]. Manuscritos: $B$ - $B r$, Ms II 3951 Mus Fétis $2430^{52}$ [RISM: 700006572]; $D$ - $B s a$, SA 1278 (14) [RISM: 469127814].

- Occhi voi v'inganate (SB). D-AN, VIg 57 [RISM: 453012072].

- Sfortunato mio cor (SC). D-AN, VIg 57 [RISM: 453012069].

- Son amante, e al cor mi sento (SC), op. III, no. 5. Impresión: I-Bc, BB.159; I-Fc, (?) [RISM A/I: P 2457].

- Stringi amor le tue ritorte (SC), op. III, no. 3. Impresión: I-Bc, BB.159; I-Fc, (?) [RISM A/I: P 2457].

- Troppo lunga dimora (SS). D-AN, VIg 57 [RISM: 453012073].

- Un tormento è la speranza (SC), op. III, no. 1. Impresión: I-Bc, BB.159; I-Fc, (?) [RISM A/I: P 2457]. Manuscritos: D-B, Mus.ms. 30257 [RISM: 455033580]; GB-Lbl, Add. 14228 [RISM: 806155086].

\section{TERCETOS:}

- Ecco il lido, a terra, a terra (STB), op. III, no. 11. Impresión: I-Bc, BB.159; I-Fc, (?). [RISM A/I: P 2457]. Manuscrito: D-B, Mus.ms. 30257 [RISM: 455033579].

- Tramonta il sol e lascia il mondo tutto (SCT), op. III, no. 12. Impresión: I-Bc, BB.159; I-Fc, (?). [RISM A/I: P 2457]. Manuscrito: D-B, Mus.ms. 30257 [RISM: 455033578].

\section{MADRIGALES:}

- Gran Dio, ah, voi languite, a cinco voces (SSCTB), Viena, 26 de febrero de $1700 .^{53} \mathrm{I}$ Baf, capsa II, n. 31 [RISM: 850016719].

\footnotetext{
52 En esta fuente el dueto está transcrito una tercera arriba (en Do menor).

53 Publicado en edición crítica en Béjar Bartolo, 2012.
} 
MisAS, O SECCIONES DE MisA: ${ }^{54}$

- Credo, para la misa del 28 de junio de 1697 (*). Música perdida.

- Credo, para la misa del 7 de julio de 1705 (*). Música perdida.

- Credo, para la misa del 7 de julio de 1711 (*). Música perdida.

- Gloria in excelsis Deo (SCB, 2 vl, bc). D-B, Mus.ms. 30257 [RISM: 455033577].

- Introito y Mottetto (Consagración), para la misa del (?) 1695 (*). Música perdida.

- Introito, para la misa del 17 de junio de 1698 (*). Música perdida.

- Introito, para la misa del 4 de julio de 1702 (*). Música perdida.

- Introito, para la misa del 13 de julio de 1706 (*). Música perdida.

- Introito, para la misa del 11 de julio de 1709 (*). Música perdida.

- Introito y Mottetto (Ofertorio), para la misa del 18 de julio de 1713 (*). Música perdida.

- Introito, para la misa del 9 de julio de 1716 (*). Música perdida.

- Kyrie y Gloria, para la misa del 19 de junio de 1708 (*). Música perdida.

- Kyrie, para la misa del (?) 1714 (*). Música perdida.

- Messa a quattro breve con VV. (= violines), 1717 (**). Música perdida.

- Mottetto (Ofertorio), para la misa del 8 de julio de 1701 (*). Música perdida.

- Mottetto (Consagración), para la misa del 5 de julio de 1712 (*). Música perdida.

\section{SALMOS, RESPONSORIOS, CÁNTICOS Y LETANÍAS:}

- Beatus, para las vísperas del 9 de julio de 1699 (*). Música perdida.

- Beatus, para las vísperas del 19 de junio de 1722 (*). Música perdida.

- Confitebor, para las vísperas del 17 de junio de 1698 (*). Música perdida.

- Confitebor, para las vísperas del 22 de junio de 1700 (*). Música perdida.

- Confitebor, para las vísperas del 5 de julio de 1712 (*). Música perdida.

54 A partir de este momento en adelante, todas las composiciones señaladas con $(*)$-hoy perdidas- fueron realizadas durante la permanencia de Pistocchi en la Academia Filarmónica de Bolonia en honor a la celebración de la fiesta del santo protector S. Antonio de Padua (Gambassi, 1992, pp. 285-289). Todas las composiciones señaladas con $(* *)$-hoy perdidas- las dejó Pistocchi al «sig. D. Domenico Zanardi, sacerdote bolognese, mio caro amico, e scolare di poco tempo" ("sacerdote boloñés, mi querido amigo y alumno desde hace poco tiempo"), como se lee en el testamento del compositor redactado el 11 de enero de 1725, confiado al notario Agostino Ignazio Pedretti (Bologna, Archivio Notarile, Augustinus Ignatius de Pedrettis, not. Instrumenta 1726, n. 106 M.): ver Busi, 1891, pp. 182-184. 
- Confitebor, a tre (SCB), 1713 (**). Música perdida.

- Confitebor, para las vísperas del 22 de junio de 1719 (*). Música perdida.

- Lauda Jerusalem (Psalmus CXLVII), a cinco voces (SSCTB) y bc (org), 1696. D-B, Mus.ms. 2755 (4) [RISM: 452004624].

- Lauda Jerusalem (Psalmus CXLVII), a cinco voces (SSCTB), orquesta (2 vl, vla, 2 ob) y bc (org). D-B, Mus.ms. 30249 (9) [RISM: 455033480].

- Laudate Dominum, para las vísperas del 26 de junio de 1703 (*). Música perdida.

- Laudate Dominum, para las vísperas del 7 de julio de 1711 (*). Música perdida.

- Laudate pueri, para las vísperas del 26 de junio de 1694 (*). Música perdida.

- Laudate pueri, para las vísperas del 28 de junio de 1697 (*). Música perdida.

- Laudate pueri, para las vísperas del 10 de julio de 1710 (*). Música perdida.

- Laudate pueri, para las vísperas del 1 de octubre de 1715 (*). Música perdida.

- Laudate pueri, para las vísperas del 9 de julio de 1716 (*). Música perdida.

- Litanie, a tre e quattro concertate, brevissime (**). Música perdida.

- Magnificat, para las vísperas del 14 de julio de 1718 (*). Música perdida.

- Miserere, a quattro, brevissimo, 1720 (**). Música perdida.

\section{HIMNOS:}

- Hymnus, para las vísperas del (?) 1695 (*). Música perdida.

- Hymnus, para las vísperas del 10 de julio de 1710 (*). Música perdida.

- Hymnus, para las vísperas del 15 de julio de 1717 (*). Música perdida.

- Tantum ergo, a due (SC), col «Genitori» a quattro, 1719 (**). Música perdida.

- Vexilla regis, a tre (SCB) con VV (= violines), 1696 (**). Música perdida.

\section{ANTÍFONAS:}

- Ave Regina calorum, per Alto solo con ripieni (**). Música perdida.

- O magnum pietatis opus, antifona a quattro per la SS. Croce (**). Música perdida.

- Salve Regina, alla capuccina con $V V$ (= violines) (**). Música perdida.

- Salve Regina, para las vísperas del 26 de junio de 1687 (*). Música perdida.

- Salve Regina, para las vísperas del 7 de julio de 1693 (*). Música perdida.

- Salve Regina, para las vísperas del 26 de junio de 1694 (*). Música perdida. 
- Salve Regina, para las vísperas del 9 de julio de 1699 (*). Música perdida.

- Salve Regina, para las vísperas del 7 de julio de 1705 (*). Música perdida.

- Salve Regina, para las vísperas del 11 de julio de 1709 (*). Música perdida.

\section{MOTETES:}

- Motete («a otto con due cori di stromenti»), ${ }^{55}$ a dos coros (I coro: SCTB, 2 vl, vla, vlc, viol, org; II coro: SCTB, 2 vl, vla, vlc, viol, org), agosto de 1703. Música perdida

- Opera Domini, mottetto a tre (SCB) (**). Música perdida.

- Opera Domini, mottetto con $V V$ (= violines) (B) (**). Música perdida.

\section{LAMENTACIONES:}

- Lettione Prima del Venerdi Santo, S y bc. I-Bof, ms 56 [RISM: 850035086].

\section{PASTORALES:}

- Pastorale, a cinque con Violini, per il S. Natale, 1721 (**). Música perdida.

\section{SECUENCIAS:}

- Victim[a]e paschali, a quattro, brevissimo (**). Música perdida.

\section{TEXTOS:}

- Texto poético para la cantata Bella rosa che vezzosa sei reina (S), musicalizada por Giuseppe Torelli. ${ }^{56}$ D-B, Mus.ms. 30212 [RISM: 455032724].

${ }^{55}$ La información referente a este motete se extrae de las tres cartas de Pistocchi enviadas (desde Pratolino) a Giacomo Antonio Perti en el verano de 1703 (18 de agosto, 25 de agosto, 8 de septiembre): Béjar Bartolo, 2014, pp. 87-94: 89, 91, 93-94.

56 En la fuente se lee: «Cantata | La poesia del Sign Pistocchi | La Compositione del Sign Torelli». El texto poético completo es el siguiente: [A1] Bella rosa, che vezzosa | sei reina d'ogni fior, | la frag[r]anza che odor[o]sa | tu respiri i martìri | non ralenta a questo cor. [R] Tu porgi al passagiero | d'ogni più grato odor il primo odore. | Ogni ninfa t'accoglie, | ogni pastor ti bacia, | l'aure istes[s]e invidiose | nel passarti rapiscono l'ardore | e a me di tanto bene | m'è vietato il piacer e le rapine, | poiché in vece d'ardor mi dai le spine. | Così Irene spietata, | im<m>itando il tuo stile e tua beltade, | niega amor per odor, e sol concede | per le spine 
- 121 cartas,$~^{57}$ dirigidas a Giovanni Paolo Colonna, Giacomo Antonio Perti $(I-B C)^{58}$ y al Margrave de Brandeburgo (¿D-AN ${ }^{59}$ o $\left.D-B A a ?\right){ }^{60}$

\section{Discusión}

El listado anterior constituye una herramienta fundamental para cualquier estudio sobre la música de Francesco Antonio Pistocchi y representa una primera aproximación a su producción global, en el sentido que dicho elenco de obras está sujeto inevitablemente a las actualizaciones constantes de los catálogos de las diferentes bibliotecas del mundo y del RISM. De cualquier manera, el listado aquí presentado ofrece una cantidad bastante relevante de información, también sobre nuevas fuentes musicales encontradas. En la descripción de cada composición resguardada se ha incluido su localización (biblioteca y signatura), pero no se ha especificado si se trata de una fuente autógrafa o copia: ${ }^{61}$ esto será el propósito de un estudio sucesivo que servirá también para establecer nuevas atribuciones eventuales a Pistocchi.

i martì in mia mercede. [A2] Troppo simile ad Irene | vago fior mostri il tuo stato; | se le spine a me tu porgi, | ella a me dona le pene, | ambi uniti al mio tormento | più severo e più spietato.

57 Según Schnoebelen. 2001, p. 791.

58 Las cartas resguardadas en $I-B c$ (http://www.bibliotecamusica.it/cmbm/scripts/lettere/lettere.asp?id=3680) han sido transcritas en Béjar Bartolo, 2014, pp. 48-98.

59 Según Dubowy, 2007, col. 638.

60 Según Schnoebelen, 2001, p. 791.

61 Entre las fuentes autógrafas hay que mencionar, por ejemplo, las del madrigal Gran Dio, ah, voi languite y de la lamentación Lettione Prima del Venerdi Santo. 


\section{Conclusiones}

Al finalizar la presente investigación, gracias a la cual ha sido posible identificar más de 150 composiciones - existentes o perdidas- de Francesco Antonio Pistocchi, se ha encontrado la música de casi 90 obras, actualmente resguardadas en alrededor de treinta bibliotecas diferentes de Alemania (Ansbach, Staatliche Bibliothek; Berlin, Staatsbibliothek y SingAkademie; Münster, Santini-Bibliothek; Sondershausen, Stadt- und Kreisbibliothek "Johann Karl Wezel”; Wolfenbüttel, Herzog August Bibliothek), Austria (Wien, Österreichische Nationalbibliothek), Bélgica (Bruxelles, Conservatoire royal de Bruxelles y Bibliothèque royale de Belgique), Francia (Paris, Bibliothèque nationale de France), Italia (Bologna, Accademia Filarmonica, Museo Internazionale e Biblioteca della Musica, Bibliotecaarchivio della Congregazione dell'Oratorio di San Filippo Neri y Archivio Musicale della Basilica di San Petronio; Firenze, Biblioteca del Conservatorio di Musica "Luigi Cherubini"; Genova, Biblioteca del Conservatorio di Musica "Niccolò Paganini"; Milano, Biblioteca del Conservatorio di Musica "Giuseppe Verdi"; Modena, Biblioteca Estense Universitaria; Napoli, Biblioteca del Conservatorio di Musica "S. Pietro a Majella"; Padova, Biblioteca Antoniana; Parma, Biblioteca Nazionale Palatina; Pavia, Biblioteca Universitaria; Torino, Biblioteca Nazionale Universitaria; Venezia, Biblioteca della Fondazione QueriniStampalia), Reino Unido (London, Royal Academy of Music, The British Library y Gresham College; Oxford, Bodleian Library) y Estados Unidos de América (Washington, D.C., The Library of Congress).

Lo anterior es el testimonio más evidente de la amplia circulación de la obra de Francesco Antonio Pistocchi, lo cual confirma el alto valor artístico de su música. ${ }^{62}$

\footnotetext{
${ }^{62}$ La contribución individual de cada autor de este artículo es la siguiente: Alejandra Béjar Bartolo (integrante del Cuerpo Académico Consolidado de "Musicología”, UGTO-CA-66, de la Universidad de Guanajuato) es la responsable de la redacción del artículo, así como del catálogo de Pistocchi referente a los géneros de cantatas, duetos, tercetos, madrigales, misas (o secciones de misa), salmos, responsorios, cánticos, letanías, himnos, antífonas, motetes, lamentaciones, pastorales y secuencias; Fabrizio Ammetto (responsable del Cuerpo Académico Consolidado de "Musicología", UGTO-CA-66, de la Universidad de Guanajuato) es el responsable de la revisión del artículo, así como del catálogo de Pistocchi referente a los géneros de óperas, serenatas, oratorios y música instrumental.
} 


\section{Bibliografía}

Basso, A. (1985-1988). Dizionario enciclopedico universale della musica e dei musicisti. Le biografie (8 vols.). Torino: UTET.

Béjar Bartolo, A. (2010). Francesco Antonio Mamiliano Pistocchi (1659-1726): contexto histórico, aspectos biográficos e inventario de su obra. Trabajo de investigación para la obtención del Diploma de Estudios Avanzados. Madrid: Universidad Autónoma de Madrid (Doctorado en Historia y Ciencias de la Música).

Béjar Bartolo, A. (2012). Francesco Antonio Mamiliano Pistocchi: Madrigale 'Gran Dio, ah, voi languite'. Edizione critica. Lucca: LIM - Libreria Musicale Italiana.

Béjar Bartolo, A. (2014). La música vocal impresa de Francesco Antonio Pistocchi (16591726): Scherzi musicali, [op. II] y Duetti e terzetti, op. III. Tesis doctoral. Guanajuato: Universidad de Guanajuato (Doctorado en Artes).

Béjar Bartolo, A. (2015). Scherzi musicali, [op. II] e Duetti e terzetti, op. III. Edizione critica. Lucca: LIM - Libreria Musicale Italiana.

Bonlini, G. C. (1730). Le glorie della poesia, e della musica contenute nell'esatta notitia de teatri della città di Venezia, e nel catalogo purgatissimo de drami musicali quivi sin hora rapresentati, con gl'auttori della poesia, e della musica, e con le annotationi a suoi luoghi proprij. Venezia: Carlo Buonarrigo.

Burney, C. (1789). A General History of Music from the Earliest Ages to the Present Period (4 vols., 1776-1789). London: Becket and others.

Busi, L. (1891). Il Padre G. B. Martini musicista-letterato del secolo XVIII. Bologna: Nicola Zanichelli.

Chiarelli, A. (1988), Pistocchi, Francesco Antonio Mamiliano, en: Basso, A. (1985-1988). Dizionario enciclopedico universale della musica e dei musicisti. Le biografie (8 vols.). Torino: UTET, vol. 6, pp. 33-34.

Dellaborra, M. y Peroni, A. (2008). «La viola gialla». Fonti musicali nella Biblioteca universitaria di Pavia. Catalogo della mostra 21 giugno - 15 settembre 2007. ComoPavia: Ibis.

Dubowy, N. (2007). Pistocchi, Francesco Antonio Mamiliano, en: Finscher, L. (1994-2007). Die Musik in Geschichte und Gegenwart: [...] zweite, neubearbeitete Ausgabe, Personenteil (17 vols.). Kassel-Stuttgart: Bärenreiter-Metzler, vol. 13, cols. 637-638. 
Finscher, L. (1994-2007). Die Musik in Geschichte und Gegenwart: [...] zweite, neubearbeitete Ausgabe, Personenteil (17 vols.). Kassel-Stuttgart: BärenreiterMetzler.

Gambassi, O. (1992). L'Accademia filarmonica di Bologna. Fondazione, statuti e aggregazioni. Firenze: Olschki.

Lora, F. (2015). Pistocchi, Francesco Antonio Mamiliano, en: Dizionario Biografico degli Italiani. Roma: Istituto della enciclopedia italiana, vol. 84, en línea: $<$ http://www.treccani.it/enciclopedia/francesco-antonio-mamilianopistocchi_(Dizionario-Biografico)/>.

Mischiati, O. (1984). Indici, cataloghi e avvisi degli editori e librai musicali italiani dal 1591 al 1798. Firenze: Olschki.

Penna, O. (1736). Catalogo degli aggregati della Accademia Filarmonica di Bologna (2 vols. manuscritos en $I-B a f)$.

Quantz, J. J. (1752). Versuch einer Anweisung die Flöte traversiere zu spielen. Berlin: Voss.

Rasch, R. (2001). The Italian Presence in the Musical Life of the Dutch Republic, en: Strohm, R. (2001). The Eighteenth-Century Diaspora of Italian Music and Musicians. Turnhout: Brepols, pp. 177-210.

Ryom, P. (2007). Antonio Vivaldi. Thematisch-systematisches Verzeichnis seiner Werke (RV). Wiesbaden: Breitkopf \& Härtel.

Sadie, S. (2001). The New Grove Dictionary of Music and Musicians, second edition (29 vols.). London: Macmillan.

Schnoebelen, A. (2001). Pistocchi, Francesco Antonio Mamiliano, en: Sadie, S. (2001). The New Grove Dictionary of Music and Musicians, second edition (29 vols.). London: Macmillan, vol. 19, pp. 790-791.

Silvani, G. A. (1724). Indice dell'opere di musica sin'ora stampate in Bologna, e si vendono da G. A. Silvani. Bologna: Giuseppe Antonio Silvani.

Tosi, P. F. (1723). Opinioni de' cantori antichi, e moderni, o sieno Osservazioni sopra il canto figurato. Bologna: Lelio dalla Volpe.

Wessel, K. (2004). Duetti da camera. Handschriftliche Sammlung aus der Schlossbibliothek Ansbach mit 18 Duetten von Navarra, Perti, Pistocchi, Steffani, Torri, und Ziani, für zwei Singstimmen \& B.c. (2 vols.). Magdeburg: Edition Walhall. 